\title{
Appearance of bound states in random potentials with applications to soliton theory
}

\author{
S. A. Derevyanko* \\ Nonlinearity and Complexity Research Group, Aston University, Aston Triangle, Birmingham B4 7ET, United Kingdom
}

(Received 27 December 2010; published 6 July 2011)

\begin{abstract}
We analyze the stochastic creation of a single bound state (BS) in a random potential with a compact support. We study both the Hermitian Schrödinger equation and non-Hermitian Zakharov-Shabat systems. These problems are of special interest in the inverse scattering method for Korteveg-de-Vries and the nonlinear Schrödinger equations since soliton solutions of these two equations correspond to the BSs of the two aforementioned linear eigenvalue problems. Analytical expressions for the average width of the potential required for the creation of the first BS are given in the approximation of delta-correlated Gaussian potential and additionally different scenarios of eigenvalue creation are discussed for the non-Hermitian case.
\end{abstract}

DOI: 10.1103/PhysRevE.84.016601

PACS number(s): 05.10.Gg, 05.45.Yv, 03.65.Ge

\section{INTRODUCTION}

The goal of this paper is to calculate the probabilities of generating soliton pulses from a random initial pulse with finite support. Two landmark soliton-bearing nonlinear systems are studied with this regard: the Korteveg-de Vries (KdV) equation and the focusing nonlinear Schrödinger equation (NLSE). Both have the remarkable property of being integrable by the so-called inverse scattering transform (IST) [1]. In this technique the number of solitons, their parameters, and asymptotic behavior at large times are completely determined by the associated linear eigenvalue problem where the initial pulse shape acts as a "potential." The discrete eigenvalues or bound states (BSs) of the corresponding linear operator (if exist) determine the properties of the soliton part of the solution while the continuous part of the spectrum is responsible for the radiation. Remarkably the corresponding linear operators occurring in the IST are those studied in various areas of quantum physics. In particular, the linear eigenvalue problem for the $\mathrm{KdV}$ equation is exactly the stationary Hermitian Schrödinger equation while for the Zakharov-Shabat (ZS) eigenvalue problem describing the properties of NLSE solitons is a non-Hermitian extension of one-dimensional (1D) Dirac and pseudogap models used in the theory of superconductivity [2-4].

Here we are interested in a specific subclass of soliton problems, namely soliton generation from a stochastic input. Already vast literature exists on the subject including a relatively recent textbook [5]. Note that, as mentioned earlier, the associated linear eigenvalue problems for both $\mathrm{KdV}$ and NLSE have relevance in the general context of quantum disordered systems and therefore the results of this paper have relevance beyond the scope of soliton theory. In the context of the latter the particular motivation behind the current paper lies in the field of nonlinear fiber optics communication where pulse propagation in optical fiber is governed by NLSE and it is important to study the process of the appearance of "ghost" soliton pulses from a purely random input as these ghost pulses lead to errors in a digital bit stream [6]. Random initial problems for $\mathrm{KdV}$ have been studied also (mostly

*s.derevyanko@aston.ac.uk numerically [7]) with regards to shallow water waves in the ocean.

Since the asymptotic soliton properties of both $\mathrm{KdV}$ and NLSE are determined by the discrete spectra of the linear Schrödinger equation and ZS operator, respectively, in the current paper it is these linear random spectra that are studied. The real discrete "energies" of the associated Schrödinger equation correspond to the (random) amplitudes of the $\mathrm{KdV}$ solitons while the real and complex parts of complex discrete eigenvalues of the corresponding ZS operator determine both the amplitudes and velocities of the NLSE solitons [1]. Here we will restrict ourselves to the problem of initial pulses (i.e., random potentials) of small finite support and calculate the average width of the potential required to create a single discrete BS (i.e., soliton) within the given interval of parameters. It turns out that the problem can be quite straightforwardly reformulated as the mean first passage problem (MFPP) of a random walker in a bounded domain and for the case of Gaussian delta correlated potential some analytic results can be obtained.

\section{HERMITIAN SCHRÖDINGER EQUATION}

We shall begin with the case of a 1D Schrödinger equation with real disordered potential $U(x)$ with finite support $[0, L]$

$$
-\psi^{\prime \prime}(x)+U(x) \psi=E \psi, \quad 0 \leqslant x \leqslant L .
$$

At this point we make no particular assumptions regarding the statistics of the potential $U(x)$. Since the potential is real the eigenfunctions can also be chosen as such. It is known that the spectral properties of the equation above determine the properties of the solution of the attached nonlinear $\mathrm{KdV}$ equation [1]

$$
U_{t}-6 U U_{x}+U_{x x x}=0
$$

where the initial condition for the $\mathrm{KdV}$ equation $U(0, x)$ plays the part of the potential in Eq. (1).

The type of boundary conditions that arises in the IST method for the $\mathrm{KdV}$ equation [1] are such that the bound states (localized Jost functions) have the prescribed boundary 
values of the logarithmic derivatives

$$
\left.\frac{\psi^{\prime}}{\psi}\right|_{x=0}=-i k \equiv z_{0}(E),\left.\quad \frac{\psi^{\prime}}{\psi}\right|_{x=L}=i k \equiv z_{L}(E),
$$

where $k=\sqrt{E}$ are imaginary wave vectors corresponding to the discrete negative energy spectrum. Each discrete energy level $E_{n}$ corresponds to a $\mathrm{KdV}$ soliton with the amplitude $-2 E_{n}$ evolving from the initial pulse shape $U(x)$.

Note that such "outgoing" boundary conditions differ from the classical Sturm-Liouville problem (SLP) where the endpoint logarithmic derivatives $z_{0}, z_{L}$ are arbitrary real constants [8-10]. Here these quantities are energy dependent and satisfy symmetry relations $z_{0}(E)=-z_{L}(E)$. Hence the spectral properties of the considered eigenvalue problem are different.

Following Ref. [9] it is convenient to introduce the real phase function assuming that $\cot \alpha(x)=\psi^{\prime}(x) / \psi(x)$. From Eq. (1) it follows that the phase $\alpha$ as a function of coordinate $x$ and a free energy parameter $E$ satisfies the following initial value problem

$$
\frac{\partial \alpha}{\partial x}=\cos ^{2} \alpha+[E-U(x)] \sin ^{2} \alpha, \quad \cot \alpha_{0}=z_{0}(E),
$$

with $0 \leqslant \alpha_{0}(E) \leqslant \pi / 2$. The most important feature of the phase $\alpha$ is that like in the SLP case [9] it is a monotonic function of energy [i.e., $\partial \alpha(x, E) / \partial E>0$ at each point $x$ ]. This can be seen by differentiating Eq. (4) with respect to $E$ and integrating the resulting linear equation.

Obviously not all the solutions of the Cauchy problem (4) will correspond to the true spectrum $E_{n}$, but only those for which $\cot \alpha\left(L ; E_{n}\right)=z_{L}\left(E_{n}\right) \equiv \cot \alpha_{L}\left(E_{n}\right)$. If we fix $\alpha_{L}(E)=\pi-\alpha_{0}(E)$ in the interval $[\pi / 2, \pi]$ then the number of energy levels in the interval $\left[E_{1}, E_{2}\right]$ is given by the number of times the function $\tilde{\alpha}(L ; E)=\alpha(L ; E)-\alpha_{L}(E)$ crosses the lines $\pi m$ where $m$ are integers. It is easy to check that $\tilde{\alpha}(L ; E)$ is also a monotonically increasing function of energy so the number of energy levels in the interval $\left[E_{1}, E_{2}\right]$ is given by

$$
N\left[E_{1}, E_{2}\right]=\left[\frac{\alpha\left(L ; E_{2}\right)-\alpha_{L}\left(E_{2}\right)}{\pi}\right]-\left[\frac{\alpha\left(L ; E_{1}\right)-\alpha_{L}\left(E_{1}\right)}{\pi}\right],
$$

where $[x]$ denotes the largest integer smaller or equal to $x$.

The result (5) is a direct analog of the level counting formula which was ingeniously used in Ref. [9] for calculating the density of states (DOS) of the SLP in the self-averaging limit $L \rightarrow \infty$. Here we would like to use this formula to another end. Namely we want to investigate the opposite limit of the short potential and study the appearance of the first bound state, which corresponds to a soliton creation by the disordered potential.

The problem of creation of a bound level can be reformulated as the problem of divergence of two random trajectories $\alpha\left(x ; E_{1}\right)$ and $\alpha\left(x ; E_{2}\right),\left(E_{1}<E_{2}\right)$. Because $\partial \alpha(x ; E) / \partial E>0$ the latter trajectory always lies above the former. Initially both trajectories start from the two values $\alpha_{0}\left(E_{1}\right)$ and $\alpha_{0}\left(E_{2}\right)$ $\left[\alpha_{0}\left(E_{1}\right)<\alpha_{0}\left(E_{2}\right)\right]$ in the interval $[0, \pi / 2]$. From Eq. (5) it follows that for finite negative values of energies $E_{1,2}$ the number of BSs in the infinitesimally short potential $L \rightarrow 0$ is equal to zero. Let us now fix some realization of random

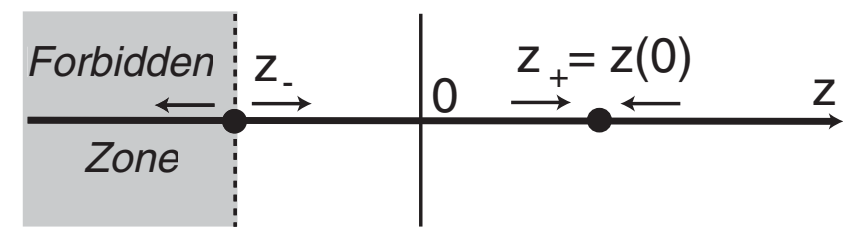

FIG. 1. The phase portrait of Eq. (6).

potential $U(x)$ and let it gradually "grow" its width. This will initiate the "dynamics" of angle trajectories according to Eq. (4). Let us denote by $l\left(E_{1}, E_{2}\right)$ the shortest occurring length $x$ when either $\alpha\left(x ; E_{1}\right)$ becomes less than $-\pi+\alpha_{L}\left(E_{1}\right)=$ $-\alpha_{0}\left(E_{1}\right)$ or $\alpha\left(x ; E_{2}\right)$ becomes greater than $\alpha_{L}\left(E_{2}\right)=\pi-$ $\alpha_{0}\left(E_{2}\right)$. According to Eq. (5) the number of bound states will become one so $l\left(E_{1}, E_{2}\right)$ has a physical meaning of a threshold for the width of potential needed for creating the first BS in the given energy interval. Since this threshold depends also on the realization of a the random potential it is in itself a random variable with its own probability density function $P\left(l ; E_{1}, E_{2}\right)$.

In what follows we will always take the limit $E_{1} \rightarrow$ $-\infty$ and put $E_{2}=E$ which gives the two trajectories that encompass all possible BSs with the energies less or equal to $E$. It easy to verify that the lower trajectory in the limit $E_{1} \rightarrow-\infty$ is just $\alpha(x ;-\infty) \equiv 0$. Therefore one only needs to consider the upper trajectory $\alpha(x ; E)$ where $\alpha(x ; E)$ evolves according to Eq. (4). A BS is then created when, for some $l(E)$, we would have $\alpha(l ; E)=\alpha_{L}(E)=\pi-\alpha_{0}(E)$.

Rather than working with the trajectory $\alpha(x ; E)$ it is convenient to go back to the original logarithmic derivative $z(x)=\cot \alpha(x)$ for which one gets the following disordered Riccati equation $[9,10]$ :

$$
z^{\prime}=-\left(z^{2}+E\right)+U(x), \quad z(0)=\sqrt{|E|},
$$

with the prime denoting differentiation with respect to $x$. The phase portrait of system (6) is given in Fig. 1. It has a stable focus $z_{+}=\sqrt{|E|}$ from which the "particle" starts initially and the unstable one at $z_{-}=-\sqrt{|E|}$ which corresponds to the creation of the first BS and, as such, acts as particle collector. In the area $z<z_{-}$the solution reaches a finite-"time" singularity $z\left(x_{*}\right)=-\infty$. However, in our problem statement the region $z<z_{-}$is forbidden since it corresponds to the trajectories beyond the threshold of creation of the first BS which are not considered in the current paper. Since the trajectory $\alpha(x ; E)$ is bound from below by the infinite energy trajectory $\alpha \equiv$ 0 this means that the particle flux at $z=\infty$ is nonpositive (i.e., the particle cannot reach $z=\infty$ moving in the positive direction of the $z$ axis). If one considers the creation of the BSs above the ground level one has to consider the trajectories in the region $z<z_{-}$and assume a continuous flux of particles from $z=-\infty$ to $z=\infty$. However, since in the first passage problem considered here such transitions are forbidden the probability flux at $z=\infty$ must be put to zero. The role of noise is to introduce a random force working against the linear positive drift $|E| z$ to bring the particle to the collector $z_{-}$. Note that the stochastic Riccati dynamics of the type above is not uncommon in many other physical applications most notably caustic formation in turbulent particle flows $[11,12]$.

Up until this point we have not prescribed particular statistics of the stochastic process $U(x)$ [i.e., the considerations 
above are applicable to any model of the field $U(x)]$. To gain further analytical insight we will from now on only consider real white Gaussian noise (WGN) with zero mean and the following correlation function $\left\langle U(x) U\left(x^{\prime}\right)\right\rangle=2 D \delta\left(x-x^{\prime}\right)$. Let us turn to the probability $G_{E}(l)$ that no BS is created for all positive widths $x$ less or equal than $l$. In terms of the stochastic trajectory $z(x)$ of Eq. (6) this is the probability that the particle starting at the point $z(0)$ will not be collected by the absorbing boundary $z_{-}$during the interval of "time" $0 \leqslant x \leqslant l$. This probability is given by

$$
G_{E}(l)=\int_{z_{-}}^{\infty} P_{E}(l ; z) d z \leqslant 1,
$$

where $P_{E}(x ; z)$ is the solution of the following Fokker-Planck equation (see standard texts $[13,14]$ for details)

$$
\begin{gathered}
\frac{\partial P_{E}}{\partial x}=-\frac{\partial J_{E}(x ; z)}{\partial z}, \\
J_{E}(x ; z) \equiv-\left(\left(z^{2}+E\right) P_{E}+D \frac{\partial P_{E}}{\partial z}\right),
\end{gathered}
$$

with the absorbing boundary condition at $z=-\sqrt{|E|}+0$ and the reflecting boundary condition at $z=\infty$

$$
P_{E}(x ;-\sqrt{|E|}+0)=0, \quad J_{E}(x ;+\infty)=0 .
$$

The initial condition for this equation for the semi-infinite energy interval is $P_{E}(0 ; z)=\delta[z-z(0)]$.

We are interested here in the probability density function for the soliton creation threshold given the initial position $z_{0}$ for which we have

$$
P\left(l ; z_{0}\right)=\frac{\partial}{\partial l}\left[1-G_{E}\left(l \mid z_{0}\right)\right]=-\frac{\partial}{\partial l} \int_{-\sqrt{|E|}}^{\infty} P_{E}(l ; z) d z .
$$

It is known that $G\left(l \mid z_{0}\right)$ satisfies the time-reversed FokkerPlanck equation with the adjoint Fokker-Planck operator $[11,13]$

$$
\frac{\partial G_{E}\left(l \mid z_{0}\right)}{\partial l}=-\left(z_{0}^{2}+E\right) \frac{\partial G_{E}\left(l \mid z_{0}\right)}{\partial z_{0}}+D \frac{\partial^{2} G_{E}\left(l \mid z_{0}\right)}{\partial z_{0}^{2}},
$$

with the initial condition $G_{E}\left(0 ; z_{0}\right)=1$ and the boundary conditions

$$
\left.G_{E}\left(l \mid z_{0}\right)\right|_{z_{0}=-\sqrt{|E|}+0}=0,\left.\quad \frac{\partial G_{E}\left(l \mid z_{0}\right)}{\partial z_{0}}\right|_{z_{0}=\infty}=0 .
$$

The general solution of this equation can be obtained by eigenvalue decomposition of the adjoint Fokker-Planck operator in the right-hand side (r.h.s.). Here we will consider only the first moment [i.e., the average potential width $l_{1}\left(z_{0}\right)$ required for creating a level which is nothing else than a mean first passage time for the particle $z(x)$ reaching the absorbing boundary]. From Eq. (9) we obtain an ordinary differential equation

$$
-1=-\left(z_{0}^{2}+E\right) \frac{d l_{1}}{d z_{0}}+D \frac{d^{2} l_{1}}{d z_{0}^{2}},
$$

subject to boundary conditions

$$
l_{1}(-\sqrt{|E|}+0)=0, l_{1}^{\prime}(\infty)=0 .
$$

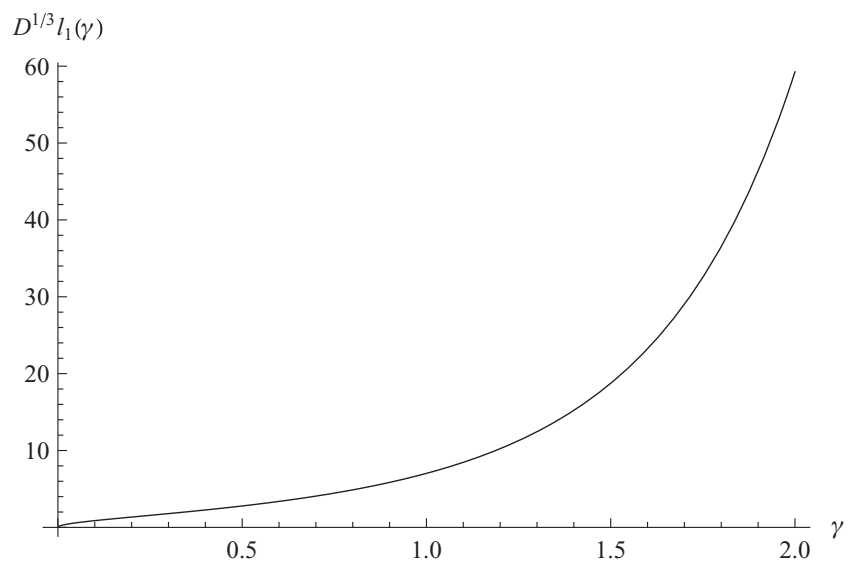

FIG. 2. The scaled average length of the potential required for creating a level below $E$ vs. the dimensionless parameter $\gamma$.

Its general solution can be obtained in quadratures and we must substitute $z_{0}=\sqrt{|E|}$ to obtain the desired soliton creation threshold. The result reads

$l_{1}=D^{-1 / 3} \gamma \int_{-1}^{1} d \eta \int_{\eta}^{\infty} d \xi \exp \left[\gamma^{3 / 2}\left(\frac{\eta^{3}-\xi^{3}}{3}+\xi-\eta\right)\right]$,

where $\gamma=|E| / D^{2 / 3}$. One can get rid of one of the integrations and write the answer as

$$
\begin{aligned}
l_{1}= & D^{-1 / 3} \frac{\sqrt{\pi}}{2} \gamma^{1 / 4} \int_{0}^{\infty} t^{-1 / 2} \exp \left[\left(-\frac{t^{3}}{12}+t\right) \gamma^{3 / 2}\right] \\
& \times\left(-\operatorname{erf}\left[\frac{1}{2}(t-2) t^{1 / 2} \gamma^{3 / 4}\right]\right. \\
& \left.+\operatorname{erf}\left[\frac{1}{2} t^{1 / 2}(2+t) \gamma^{3 / 4}\right]\right) d t
\end{aligned}
$$

In Fig. 2 we plot the quantity $D^{1 / 3} l_{1}$ versus parameter $\gamma$. Note that in the limit $\gamma \rightarrow 0$ the average length of the potential also goes to zero. This is in line with the known result from quantum mechanics that every finite $1 \mathrm{D}$ potential well, no matter how narrow, has at least one BS. In the opposite limit $\gamma \gg 1$ we have $l_{1} \propto \exp \left[(1 / 3) \gamma^{3 / 2}\right]$, that is, the average length of the potential needed to create a deep energy level (high power soliton) is exponentially large. Also note that the quantity $l_{1}$ calculated above is not the same as the average internodal distance derived in Ref. [10] for the SLP with the Dirichlet boundary conditions $\left(\alpha_{0}=\alpha_{L} \equiv 0\right)$ in the limit $L \rightarrow$ $\infty$, although both quantities seem to have similar exponential behavior in the limit $|E| \rightarrow \infty$.

\section{ZAKHAROV-SHABAT EIGENVALUE PROBLEM}

Lets us now move to the NLSE. The focusing NLSE in the normalized units has the form

$$
i q_{t}+\frac{1}{2} q_{x x}+|q|^{2} q=0 .
$$

If the initial shape of the solution is $q(0, x)=Q(x)$ then the properties of the emerging solitons are determined by the 
following nonself-adjoint problem [1]:

$$
\left\{\begin{array}{l}
i \partial \varphi_{1} / \partial x+Q(x) \varphi_{2}=\zeta \varphi_{1}, \\
-i \partial \varphi_{2} / \partial x-Q^{*}(x) \psi_{1}=\zeta \varphi_{2},
\end{array}\right.
$$

where $Q(x)$ is now a complex disordered potential and we again assume that it has a finite support $[0, L]$. The problem above can be viewed as a nonself-adjoint generalization of a 1D Dirac equation [2,9] where one has a complex energy gap $Q$ [4]. Since this eigenvalue problem is nonself-adjoint its discrete eigenvalues (BSs) are complex $\zeta_{n}=\xi_{n}+i \eta_{n}, \eta_{n}>0$ and in soliton theory, each discrete eigenvalue corresponds to a soliton component emerging form the initial field distribution $Q(x)$. The real part of each discrete eigenvalue $\xi_{n}$ provides soliton velocity while the imaginary part $\eta_{n}$ is responsible for the amplitude of a soliton.

The problem of the creation of random discrete BSs of Eq. (14) was studied in Ref. [15] while in Ref. [16] the density of such states was calculated in the self-averaging limit $L \rightarrow$ $\infty$. One of the results of Ref. [15] was that no solitons can possibly be created in the case of white Gaussian potential; a result that seems to be at odds with the finite DOS obtained in Ref. [16]. Therefore we shall later discuss the origin of such a discrepancy.

We start with the boundary conditions for system (14). It follows from IST [1] that for the finite support potential the solutions $\Phi(x ; \zeta)=\left(\varphi_{1}, \varphi_{2}\right)^{T}$ satisfy the following effective boundary conditions at the ends of the support interval:

$$
\Phi(0 ; \zeta)=\left(\begin{array}{l}
1 \\
0
\end{array}\right), \quad \Phi(L ; \zeta)=b(\zeta) e^{i \zeta L}\left(\begin{array}{l}
0 \\
1
\end{array}\right),
$$

where $b(\zeta)$ is the second Jost coefficient. As with the Hermitian case considered previously it is desirable to reduce the boundary value problems (14) and (15) to a random initial value problem (which constitutes the so-called invariant imbedding method [5]). To this end one can consider the function $r(x ; \zeta)=\varphi_{2}(x ; \zeta) / \varphi_{1}(x ; \zeta)$. It is (up to a phase factor) a reflection coefficient of a system of size $x$ and if $\varphi_{1,2}$ evolve according to Eq. (14) the function $r$ evolves according to the following random Riccati equation $[5,15]$ :

$$
\frac{d r}{d x}=2 i \zeta-i Q r^{2}+i Q^{*}, \quad r(0 ; \zeta)=0 .
$$

The difference of Eq. (6) from that of Refs. $[5,15]$ is that we permit all values of the parameter $\zeta=\xi+i \eta$ in the upper complex half-plane $\eta \geqslant 0$. Obviously not all possible values of $\zeta$ will yield an evolution of a BS but only those for which $\varphi_{1,2}$ satisfy the second boundary condition in Eq. (15), which means that for a true BS with eigenvalue $\zeta_{n}$ the quantity $r$ is singular at the boundary $\left|r\left(L ; \zeta_{n}\right)\right|=\infty$.

Working with singular variables is not always convenient so one can introduce angular variables [similar to phase $\alpha(x ; E)$ in the Hermitian Schrödinger case] using the parametrization from Ref. [15] $r=\cot (\theta / 2) e^{i \varphi}$. Then the two angles satisfy the following equations (cf. Eq. (15) of Ref. [15], and the prime denotes differentiation with respect to $x$ ):

$$
\begin{gathered}
\theta^{\prime}=2 \eta \sin \theta-2 \operatorname{Im}\left[Q e^{i \varphi}\right], \quad \theta(0)=\pi, \\
\varphi^{\prime}=2 \xi-2 \cot \theta \operatorname{Re}\left[Q e^{i \varphi}\right],
\end{gathered}
$$

where the initial value of the phase $\varphi(0)$ is chosen so that $\operatorname{Re}\left[Q(0) e^{i \varphi(0)}\right]=0$ and the initial rate of change of the phase $\varphi$ is finite. The solution of Eq. (17) at the end of the interval $\theta(L ; \zeta)$ plays the role of the level counting angle $\alpha(L ; E)$ from Eq. (5). Namely, the number of discrete complex eigenvalues $\zeta_{n}$ (and hence the number of BSs and solitons) contained inside a closed area $\zeta \in \mathcal{D}$ of the upper complex half-plane is given by the number of times the real-valued function of a complex argument $\theta(L ; \zeta) \equiv \theta_{L}(\xi, \eta)$ crosses the levels $2 \pi m$ while $\zeta$ runs through the whole of $\mathcal{D}$ ( $m$ being an integer). The situation now is much more complex than in the Hermitian case because(i) one now has to work with the function of two variables rather than one and (ii) the function $\theta_{L}(\xi, \eta)$ is generally nonmontonic (i.e., it may have multiple maxima and minima as well as saddle points). There are, however, two cases where the dynamics of the two angular variables decouples and the problem becomes 1D again. The first case is when one considers the so-called breather states (i.e., the ones where $\xi=0$ and $\zeta_{n}=i \eta_{n}$ ) in an arbitrary real potential $q(x)$ (random or deterministic) while the second one is the case where $Q(x)$ is a complex WGN with zero average and the only nonzero binary correlation function $\left\langle Q^{*}\left(x^{\prime}\right) Q(x)\right\rangle=2 D \delta\left(x-x^{\prime}\right)$. In the former case the phase $\varphi$ is stationary $\varphi= \pm \pi / 2$ and the evolution of the angle $\theta$ is given by

$$
\theta^{\prime}(x)=2 \eta \sin \theta \pm 2 q(x), \quad \theta(0)=\pi .
$$

We will choose a WGN model for our real potential $\left\langle q(x) q\left(x^{\prime}\right)\right\rangle=D \delta\left(x-x^{\prime}\right)$. One can consider only the solution with the plus sign since the other one is obtained by the angle reflection $\theta \rightarrow 2 \pi-\theta$. In soliton theory this case describes breathers: localized soliton complexes oscillating around the common center of mass.

In the case of the complex WGN system (17), Eq. (18) is a system with multiplicative white noise and as such it is ambiguous and can be understood in either the Ito or Stratonovich sense $[13,14]$. Mathematically, both approaches are equally valid and produce different statistics of the dynamical random variables. The ambiguity stems from the idealized nature of white noise, and when choosing between the models one needs to employ extra physical arguments and intuition. For example, Stratonovich interpretation naturally arises when one considers a Gaussian process with a symmetric correlation function with the correlation length $l$ being the smallest scale in the system. As we shall see below, the Ito and Stratonovich models do produce cardinally different results concerning the probability of the creation of BS in system (14).

One may notice that the noise terms in Eqs. (17) and (18) are just the unitary transform of the original complex WGN: $Q \rightarrow Q \exp (i \varphi)$. If the Ito convention is adopted than this transformed noise has exactly the same statistics as the original one and the equation for the angle $\theta$ decouples from that for $\varphi$, and moreover, it is exactly equivalent to the equation for the real WGN, Eq. (19). If, on the other hand, the Stratonovich convention is adopted the statistics of the angle $\theta$ also decouples, but an extra drift term appears

$$
\theta^{\prime}(x)=2 \eta \sin \theta+2 D \cot \theta+2 q(x), \quad \theta(0)=\pi .
$$

Therefore for both real and complex WGN the stochastic dynamical problem becomes 1D as in the Hermitian case. 
Unfortunately the solution $\theta_{L}(\eta)$ in both cases (19) and (20) is not a monotonic function of $\eta$. However, one can introduce a new function $v(x)=-\cos \theta(x)$ that is, in fact, a monotonically increasing function of $\eta$ provided that no BSs are created by this trajectory. This can be checked in the same way as it was done before in the Hermitian case by differentiating the dynamical equation with respect to $\eta$ and integrating the resulting equation over $x$. If $\eta \rightarrow \infty$, the trajectory tends to the stationary state $v(x) \equiv 1$ [i.e., $\theta(x) \equiv \pi$ ] and all other trajectories yield lower values of $v$ for the finite positive values of $\eta$. The bounds of soliton creation $\theta=0,2 \pi$ both correspond to the value $v=-1$ so we can conclude that if for a given value of $\eta$ and given realization of the disordered potential the trajectory $\theta(x)$ which is a solution of either Eq. (19) or Eq. (20) remains inside the interval $[0 ; 2 \pi]$ [which means that $v(x)>-1]$ then all the trajectories with larger values of parameter $\eta$ (evaluated for the same realization) will also lie inside the interval $[0 ; 2 \pi]$ [i.e., will also have values $v(x)>-1]$. Hence the probability $G_{\eta}(l)$ that no BSs with the values of $\eta$ in the interval $[\eta ; \infty)$ are born as the length of the disordered potential grows from zero to $l$ is equal to the probability that a solution $\theta(x)$ remains within the interval $[0 ; 2 \pi]$ during the evolution of $x$ from zero to $l$. Note that once the first BS is created the function $v(x)$ is no longer monotonic and the reasoning above is not applicable for estimating the higher-level creation distance and one has to resort to the original Eqs. (19) and (20).

Let us treat the case of the real potential (or Ito WGN) first. Similar to the Hermitian case we can write down the backward Fokker-Planck equation for the probability that the level creation threshold is not reached for the length $l$ if the trajectory starts at the initial point $\theta_{0}$

$$
\frac{\partial G_{\eta}\left(l \mid \theta_{0}\right)}{\partial l}=2 \eta \sin \theta_{0} \frac{\partial G_{\eta}\left(l \mid \theta_{0}\right)}{\partial \theta_{0}}+2 D \frac{\partial^{2} G_{\eta}\left(l \mid \theta_{0}\right)}{\partial \theta_{0}^{2}},
$$

with $G_{\eta}\left(0 \mid \theta_{0}\right)=1$. Since both $\theta=0$ and $\theta=2 \pi$ are absorbing boundaries (they both correspond to the creation of a BS) one will have $G_{\eta}(l \mid 0)=G_{\eta}(l \mid 2 \pi)=0$. Solving the above for $\eta=0$ and putting $\theta_{0}=\pi$ we obtain the probability for $n o t$ creating a single BS with an arbitrary positive value of $\eta$, $G_{0}(l \mid \pi)$, which can be expressed as

$$
G_{0}(l \mid \pi)=\frac{2}{\pi} \sum_{n=0}^{\infty} \frac{(-1)^{n}}{(n+1 / 2)} \exp \left[-2 D l(n+1 / 2)^{2}\right] .
$$

The probability density function for the threshold of soliton creation is obtained from above by the simple differentiation $P(l)=-\partial G_{0} / \partial l$.

If we keep $\eta$ finite we can study the average width $l_{1}$ needed to create a BS in the region $[\eta, \infty)$. Similarly to Eq. (11) it satisfies

$$
-1=2 \eta \sin \theta_{0} \frac{d l_{1}}{d \theta_{0}}+2 D \frac{d^{2} l_{1}}{d \theta_{0}^{2}},
$$

with $l_{1}(0)=l_{1}(2 \pi)=0$. To get the correct answer the solution of the boundary problem above must be evaluated at $\theta_{0}=\pi$. The result reads

$$
l_{1}=\frac{1}{2 D} \int_{0}^{\pi} d \theta^{\prime} \int_{0}^{\theta^{\prime}} d \theta^{\prime \prime} \exp \left[\frac{\eta}{D}\left(\cos \theta^{\prime \prime}-\cos \theta^{\prime}\right)\right] \text {. }
$$

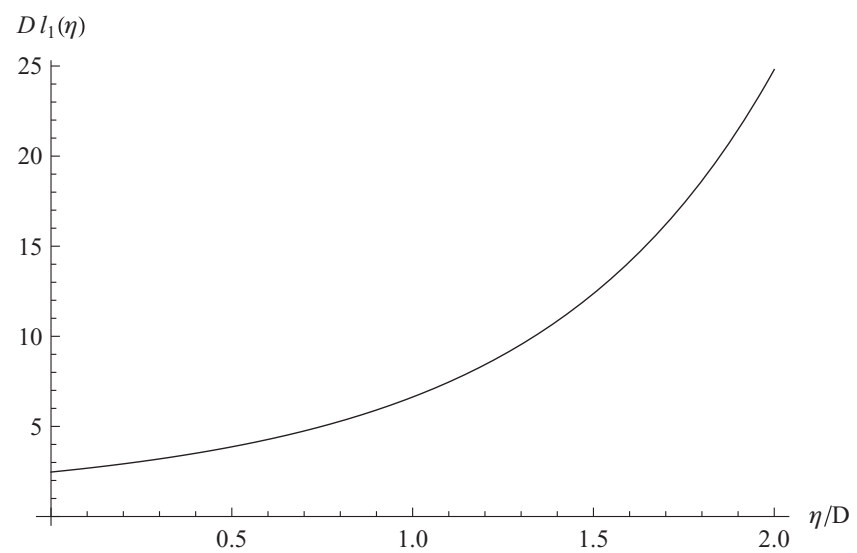

FIG. 3. The scaled average length of potential required for creating a level above $\eta$ vs. the dimensionless parameter $\eta / D$.

The dependence of the quantity $D l_{1}$ on parameter $\eta / D$ is given in Fig. 3.

Figure 3 looks not at all dissimilar to that for the Hermitian case Fig. 2. The major difference is in the limits of $\eta \rightarrow 0$ and $|E| \rightarrow 0$ correspondingly [i.e., the threshold for creating an arbitrary level (soliton)]. In the Hermitian case this threshold is always zero, while in the nonself-adjoint Zakharov-Shabat eigenvalue problem for the real potential (as well as complex Ito WGN) there is a nonzero average soliton creation threshold given by $l_{1}(0)=\pi^{2} / 4 D$.

Finally let us turn to the Stratanovich WGN case. The dynamics of the angular variable $\theta$ is given now by Eq. (20). We will here only consider the case $\eta=0$, that is, the creation of an arbitrary level (i.e., a soliton with any amplitude and velocity). Note that the extra drift term appearing in the Stratanovich picture is singular at the soliton creation boundaries $\theta=0,2 \pi$. It can be demonstrated using the Feller boundary classification [14] that these boundaries are entrance boundaries and as such cannot be reached for any finite length $x=l$. And indeed in Ref. [15] it was demonstrated (for the case $\eta=0$ ) that the corresponding Fokker-Planck equation does not have solutions with nonvanishing probability flux at the boundaries $\theta=0,2 \pi$. In other words, for the Stratanovich case not a single BS can be created by a potential of finite support. However, the stationary solution of the corresponding Fokker-Planck equation does exist and this stationary distribution is

$$
P_{s t}(\theta)=\frac{\eta}{2 D} \frac{\sin \theta \exp [-(\eta / D) \cos \theta]}{\sinh [\eta / D]}, \quad \theta \in[0, \pi] .
$$

If one makes the substitution considered earlier $v=-\cos \theta$ and additionally rescales $\tilde{D}=2 D$ the stationary distribution above matches exactly the one found in Ref. [16].

The nonexistence of BSs seems to be at odds with the results of Ref. [16] where nonvanishing DOS was obtained for a Stratonovich WGN in the limit $L \rightarrow \infty$. Therefore we shall now spend some time and elucidate the nature of such a discrepancy. In Ref. [16] the DOS for system (14) was obtained by using a formula taken from Ref. [17]

$$
\left\langle\rho\left(\zeta, \zeta^{*}\right)\right\rangle \equiv\left\langle\frac{1}{L} \sum_{k} \delta\left(\xi-\xi_{k}\right) \delta\left(\eta-\eta_{k}\right)\right\rangle=\frac{1}{\pi L} \frac{\partial}{\partial \zeta^{*}}\langle\operatorname{Tr} \mathcal{G}\rangle
$$


where $\partial / \partial \zeta^{*} \equiv(1 / 2)(\partial / \partial \xi+i \partial / \partial \eta)$ and $\mathcal{G}$ is the Green function (resolvent) of the Zakharov-Shabat eigenvalue problem. The average trace of the Green function was calculated in Ref. [16] via the Lyapunov exponent of system (14), but this is not strictly necessary. One can average the Green function directly and arrive at the same result (in the limit $L \rightarrow \infty$ )

$$
\rho\left(\zeta, \zeta^{*}\right)=\frac{1}{\pi D} \frac{(\eta / D) \operatorname{coth}(\eta / D)-1}{\sinh ^{2}(\eta / D)} .
$$

However, in the derivation of Eq. (26) an important subtlety was missed. Namely, when considering a stationary distribution at $L \rightarrow \infty$ it was automatically assumed that one can interchange the order of ensemble averaging $\langle\ldots\rangle$ and differentiation $\partial / \partial \zeta^{*}$. But as we have seen from the results of the current paper if one considers an arbitrary realization of Stratanovich WGN potential then with probability 1 no discrete eigenvalues of eigenproblem (14) occur, which means that the trace of the Green function is an analytical function everywhere above the real axis and $\partial / \partial \zeta^{*} \int \operatorname{Tr} \mathcal{G}(x, x) d x=0$ for each realization. Taking the limit $L \rightarrow \infty$ and additional ensemble averaging does not change this result. Therefore in this particular case we can say that

$$
\begin{aligned}
& \lim _{L \rightarrow \infty}\left\langle\frac{1}{L} \frac{\partial}{\partial \zeta^{*}} \int_{0}^{L} \operatorname{Tr} \mathcal{G}(x, x) d x\right\rangle \\
& \quad \neq \frac{\partial}{\partial \zeta^{*}}\left\langle\lim _{L \rightarrow \infty} \frac{1}{L} \int_{0}^{L} \operatorname{Tr} \mathcal{G}(x, x) d x\right\rangle .
\end{aligned}
$$

In other words the stationary average of an analytic function is not, in this case, analytic at any point of the upper complex plane. How does this come about? In the random matrix theory of Ref. [17] one can always interchange the ensemble averaging and differentiation over $\zeta^{*}$ because the probability measure over which the averaging is performed is fixed and does not depend on the spectral parameter $\zeta$. But in our case the probability measure used for averaging is the stationary distribution (25). It is clear from the look of Eq. (25) that this measure is (i) $\zeta$ dependent and (ii) is clearly nonanalytic since it depends only on $\eta=\left(\zeta-\zeta^{*}\right) / 2 i$. Therefore averaging over the $\zeta^{*}$-dependent measure and differentiation over $\zeta^{*}$ are not interchangeable, which explains the discrepancy between the results of the present paper (and also Ref. [15]) and the nonvanishing DOS presented in Ref. [16]. The nonexistence of BSs obtained in the present paper holds, of course, only under the idealized conditions of Stratonovich white noise [i.e., the limit of infinitely narrow, symmetrically correlated potential $Q(x)$ ]. When colored-noise potentials are considered this result may, of course, no longer hold so that there will be a nonzero probability of creating a BS by a correlated potential of finite support. However, this issue requires a more detailed analysis in each particular case, which is beyond the scope of this paper.

\section{CONCLUSION}

To sum up, we have considered a problem of creation of a single soliton from a random input of finite support in the framework of KdV and NLSE equations. Mathematically the problem amounts to finding the probability of creating a single discrete level of an either Hermitian (KdV) or non-Hermitian (NLSE) prescribed linear operator. We have shown that the latter problem can be subsequently reformulated as a stochastic first passage problem for a certain random trajectory (or rather a problem of divergence of two random trajectories) where the length of the potential plays the role of "time" in the spirit of the invariant imbedding method. In the case of Gaussian white noise the problem admits analytical treatment and the average length of the pulse needed for creating the first discrete level (soliton) was calculated for both cases. This has an important implication for fiber-optical transmission lines when a corrupted random noise propagating in and empty bit slot can develop into a "ghost" soliton pulse thus garbling the information signal. Therefore Eq. (24) (and Fig. 3) supply important information on how the average threshold of the width of the disordered pulse required for generating a ghost soliton depend on the strength of the disorder. Also the mathematical analogy with both Hermitian and non-Hermitian quantum mechanics makes the results of the current paper applicable to entirely different stochastic physical models not necessarily related to soliton propagation (e.g., Refs. [4,9]).

\section{ACKNOWLEDGMENTS}

The author wishes to thank Sergey Gredeskul and Jaroslaw Prilepsky for insightful discussions.
[1] S. Manakov, S. Novikov, L. Pitaevskii, and V. Zakharov, Theory of Solitons (Consultants Bureau, New York, 1984).

[2] A. A. Ovchinnikov and N. S. Erikhman, Sov. Phys. JETP Lett. 25, 180 (1977).

[3] E. N. Bratus', S. A. Gredeskul, L. A. Pastur, and V. S. Shumeiko, Theor. Mat. Phys. 76, 945 (1988).

[4] L. Bartosch, Ann. Phys. (Leipzig) 10, 799 (2001).

[5] V. Konotop and L. Vázquez, Nonlinear Random Waves (World Scientific, Singapore, 1994).

[6] S. K. Turitsyn and S. A. Derevyanko, Phys. Rev. A 78, 063819 (2008).

[7] E. Pelinovsky and A. Sergeeva, Eur. J. Mech. B. Fluid 25, 425 (2006).

[8] B. Halperin, Phys. Rev. 139, A104 (1965).
[9] I. Lifshitz, S. Gredeskul, and L. Pastur, Introduction to the Theory of Disordered Systems (Wiley, New York, 1988).

[10] C. Texier, J. Phys. A 33, 6095 (2000).

[11] V. I. Klyatskin and D. Gurarie, Usp. Fiz. Nauk. 169, 171 (1999).

[12] S. Derevyanko, G. Falkovich, K. Turitsyn, and S. Turitsyn, J. Turbul 8, 16 (2007).

[13] H. Risken, The Fokker-Planck Equation (Springer, Berlin, 1984).

[14] C. W. Gardiner, Handbook of Stochastic Methods (Springer, Berlin, 2004).

[15] S. A. Derevyanko and J. E. Prilepsky, Phys. Rev. E 78, 046610 (2008).

[16] P. Kazakopoulos and A. L. Moustakas, Phys. Rev. E 78, 016603 (2008).

[17] J. Feinberg and A. Zee, Nucl. Phys. B 504, 579 (1997). 